EPJ Web of Conferences 19, 03002 (2012)

DOI: $10.1051 /$ epjconf/20121903002

(C) Owned by the authors, published by EDP Sciences, 2012

\title{
The puzzling assembly of the Milky Way halo - contributions from dwarf Spheroidals and globular clusters
}

\author{
A. Koch ${ }^{1, a}$, S. Lépine ${ }^{2}$ and Ş. Çalışkan ${ }^{1,3}$ \\ ${ }^{1}$ Zentrum für Astronomie der Universität Heidelberg, Landessternwarte, Königstuhl 12, \\ 69117 Heidelberg, Germany \\ ${ }^{2}$ Department of Astrophysics, Division of Physical Sciences, American Museum of Natural \\ History, Central Park West at 79th Street, New York, NY 10024, USA \\ ${ }^{3}$ Department of Astronomy and Space Sciences, Ankara University, 06100 Tandoğan, \\ Ankara, Turkey
}

\begin{abstract}
While recent sky surveys have uncovered large numbers of ever fainter Milky Way satellites, their classification as star clusters, low-luminosity galaxies, or tidal overdensities remains often unclear. Likewise, their contributions to the build-up of the halo is yet debated. In this contribution we will discuss the current knowledge of the stellar populations and chemo-dynamics in these puzzling satellites, with a particular focus on dwarf spheroidal galaxies and the globular clusters in the outer Galactic halo. Also the question of whether some of the outermost halo objects are dynamically associated with the (Milky Way) halo at all is addressed in terms of proper measurements in the remote Leo I and II dwarf galaxies.
\end{abstract}

\section{INTRODUCTION}

[1]'s picture of a hierarchical assembly of galaxies like the Milky Way (MW) has been bolstered by the discoveries of large numbers of ever fainter satellites around the MW and M31 in recent, ambitious sky surveys. These systems range from relatively luminous dwarf spheroidal (dSph) galaxies towards ever fainter objects, commonly dubbed ultrafaint dwarfs (UFDs; e.g., [2-6]; see also [7], and references therein). At $10^{3}-10^{5} \mathrm{M}_{\odot}$, the stellar masses of the UFDs are comparable to the most extended MW star clusters. Intriguingly, those globular clusters (GCs) with the largest radii, in the transition regime between GCs and UFDs (e.g., Fig. 1 in [8]), are predominantly found in the outermost MW halo ${ }^{1}$. In fact, current scenarios envision a dichotomy of an inner halo, formed in situ, and an outer, accreted component. In the following we will tackle the "puzzle" of the Galactic halos - the formation history of the entirety (read: the MW halo) - by studying its complexity of constituents (i.e., its halo GCs and dSph satellites). In particular, we address the discrimination between UFDs and the extended outer halo GCs and their role in assembling the Galactic halo.

\section{WINNOWING DSPHS FROM STAR CLUSTERS}

DSph galaxies have always been characterized as low-luminosity systems (see reviews by [7]; [9]). Some noteworthy key features of the dSphs are, amongst others, their low luminosities, their high dark matter content (with mass-to-light ratios, $M / L$, of up to several thousands), the omnipresence of old

\footnotetext{
ae-mail: akoch@lsw.uni-heidelberg.de

${ }^{1}$ Those clusters are also typically younger than inner halo clusters with otherwise comparable properties.
}

This is an Open Access article distributed under the terms of the Creative Commons Attribution-Noncommercial License 3.0, which permits unrestricted use, distribution, and reproduction in any noncommercial medium, provided the original work is properly cited. 

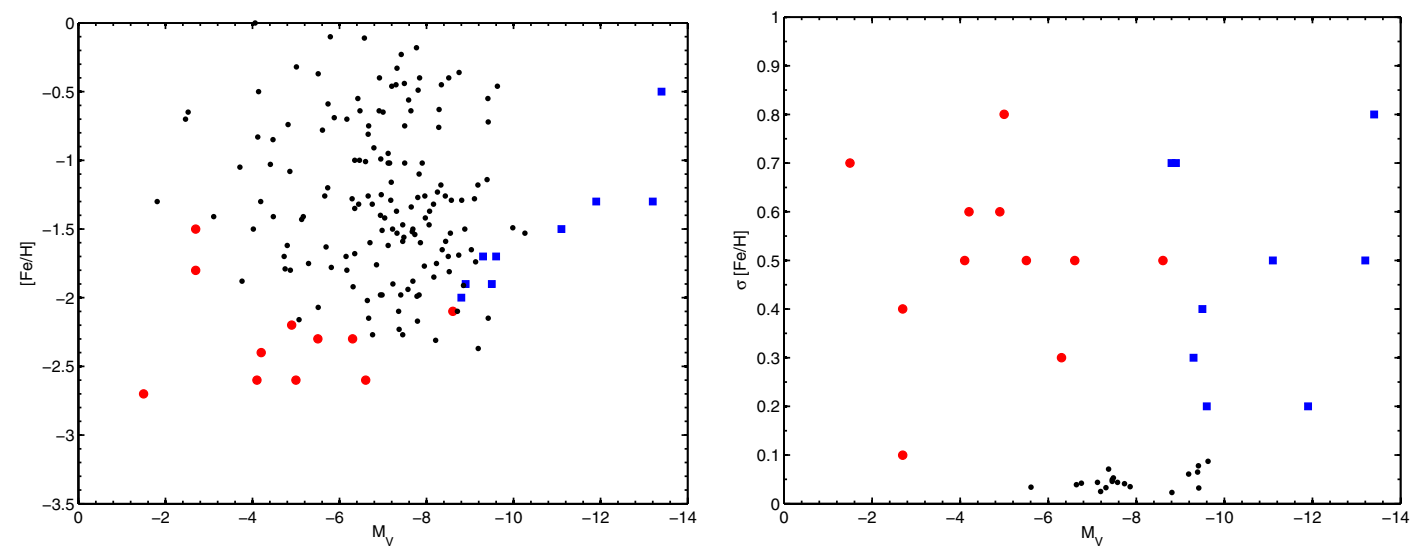

Figure 1. Mean metallicity (left panel) and $1 \sigma$ spreads (right panel) of Galactic GCs (black dots), the classical dSphs (blue symbols), and ultrafaint dSphs (red points); using data from [7] (and references therein); [12] [2011 version]; and [13].

(>12 Gyr) stellar populations, low metallicities, a slow chemical evolution, and generally complex star formation histories ([9], [10]). However, for some objects it is yet unclear whether they are truly old and metal poor systems like the dSphs, or very extended, (tidally) perturbed stellar systems, and thus essentially dying star clusters, free of dark matter, or mere density enhancements in tidal streams. In the latter cases, the "missing satellite problem" would remain a problem (e.g., [11]).

The dSphs have only experienced slow chemical evolution and little chemical enrichment, rendering them metal poor systems. As Fig. 1 (left panel) shows, they follow a well defined metallicity-luminosity relation that extends down to the faintest galaxies. The simple reason for such a correlation is that the dSphs possess deep (dark matter) potential wells, in which gas can be efficiently retained for further enrichment. GCs, on the other hand, are dark matter free and no such relation exists. They rather cover a broad range of metallicities ${ }^{2}$ irrespective of their luminosity: a low metallicity alone does not signify a dSph.

On the other hand, the deep potentials of the dSphs enable prolonged star formation and enrichment of subsequent generations of stars with the retained metals of the previous generations. As a consequence, the dSphs exhibit abundance spreads of several tenths of a dex, which is in clear contrast to the GCs that are, to first order, considered mono-metallic ${ }^{3}$ (Fig. 1, right panel).

We mention here two prime examples, for which a clear-cut classification has been controversial since their discoveries. Firstly, it has been suggested that the faint object Segue 1 could be a dissolving star cluster, associated with the Sagittarius (Sgr) dwarf; overlap (on the sky and in radial velocity) would lead to an inflated velocity dispersion so that the inferred high $M / L$ fails as an unambiguous indicator of a dark matter dominated dSph ([15]). Subsequently, [16] measured $M / L \sim 3400$, which is not explicable by contamination with Sgr stars alone. Segue 1 has a low, mean iron abundance of -2.7 dex and a $1 \sigma$ iron spread of 0.7 dex. Moreover, the full abundance ranges, e.g., in $[\mathrm{C} / \mathrm{H}]$ and $[\mathrm{Fe} / \mathrm{H}]$ are in excess of 1.5 dex, thus spanning a factor of several tens in the (heavy) element content ([17]; see also the contribution by G. Gilmore in this Volume), pointing to a dark matter dominated system (i.e., the potential well was deep enough to allow for chemical self-enrichment).

\footnotetext{
${ }^{2}$ Also note that star clusters do not contain any very metal poor stars below $\sim-2.4$ dex.

${ }^{3}$ Currently, evidence for multiple generations of stars in GCs is accumulating and some light chemical elements are found to vary within any given cluster due to their specific internal evolutionary and enrichment histories. For instance, [14] suggested the presence of a $\mathrm{Na}$-O anti-correlation as a defining factor for a "GC".
} 


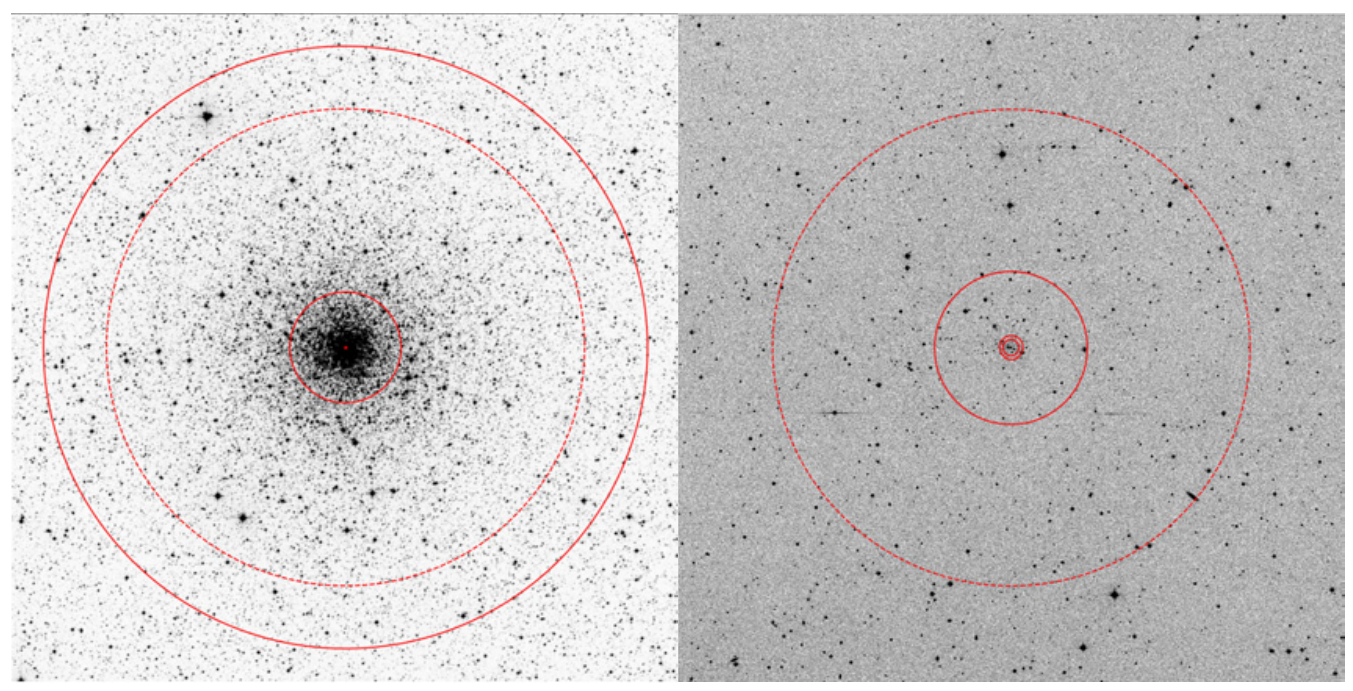

Figure 2. DSS images ${ }^{4}$ of the core-collapsed $\left(\mathrm{r}_{c}=0.03 \mathrm{pc} ; \mathrm{r}_{h}=3 \mathrm{pc} ; \mathrm{r}_{t}=16 \mathrm{pc}\right)$, close $\left(\mathrm{R}_{\odot}=2.3 \mathrm{kpc}\right)$ GC NGC 6397 (left panel), and the remote $\left(\mathrm{R}_{\odot}=93 \mathrm{kpc}\right)$, extended $\left(\mathrm{r}_{c}=11 \mathrm{pc} ; \mathrm{r}_{h}=16 \mathrm{pc} ; \mathrm{r}_{t}=108 \mathrm{pc}\right)$ cluster Pal 3 (right panel). Solid lines indicate the aforementioned radii, while the dashed circle shows the field of view of the FLAMES spectrograph (25' diameter). Each image extends $17^{\prime} \times 17^{\prime}$.

Another example of this class is Boötes II ([4]; [18]): based on low-resolution spectra of 5 memberstars, the latter work finds a mean metallicity and radial velocity dispersion consistent with a dark matter dominated, old, and metal poor dSph-like population; however, Boötes II lies square on the leading arm of Sgr in projected location on the sky, radial velocity, and distance. Every possible chemical abundance information, ideally for large numbers of stars, is thus required to describe the chemical evolution of such objects to relate them to the earliest galactic enrichment phases and to assess their role to the build-up of the halo.

\section{OUTER HALO GCS}

As elaborated above, ideally, we need to monitor the chemical abundance patterns and search for spreads in the faint structures to fully characterize their nature. Likewise, the chemical abundance patterns of GCs in the outer MW halo bears vital information about the formation and assembly history of the Galaxy. The need to obtain spectroscopy of single stars in the remote GCs of the outer halo (say, $>30 \mathrm{kpc}$ ) suffers from two main problems: At a first glance, these systems appear sparse and they are, on average, spatially more extended than GCs of the inner halo (e.g., [19]). Naïvely, this seems ideal to easily obtain uncontaminated spectroscopy for statistically significant samples of stars, preferably in multi-object mode. As the example of an inner halo cluster, NGC 6397 (at $R_{\odot}=2.3 \mathrm{kpc}$ ), in Fig. 3 (left panel) shows, this is common practice and these objects are spectroscopically well studied (e.g.,

\footnotetext{
${ }^{4}$ Copyright Note: Based on photographic data obtained using The UK Schmidt Telescope. The UK Schmidt Telescope was operated by the Royal Observatory Edinburgh, with funding from the UK Science and Engineering Research Council, until 1988 June, and thereafter by the Anglo-Australian Observatory (AAO). Original plate material is copyright $\odot$ of the Royal Observatory Edinburgh and the AAO. The plates were processed into the present compressed digital form with their permission. The Digitized Sky Survey was produced at the Space Telescope Science Institute under US Government grant NAG W-2166. Plates from this survey have been digitized and compressed by the STScI. The digitized images are copyright @ 1993-2000 by the AAO Board, and are distributed herein by agreement. All Rights Reserved. All material not subject to the above copyright provision is copyright (C) 2000 by the Association of Universities for Research in Astronomy, Inc. All Rights Reserved. Produced under Contract No. NAS5-2555 with the National Aeronautics and Space Administration.
} 


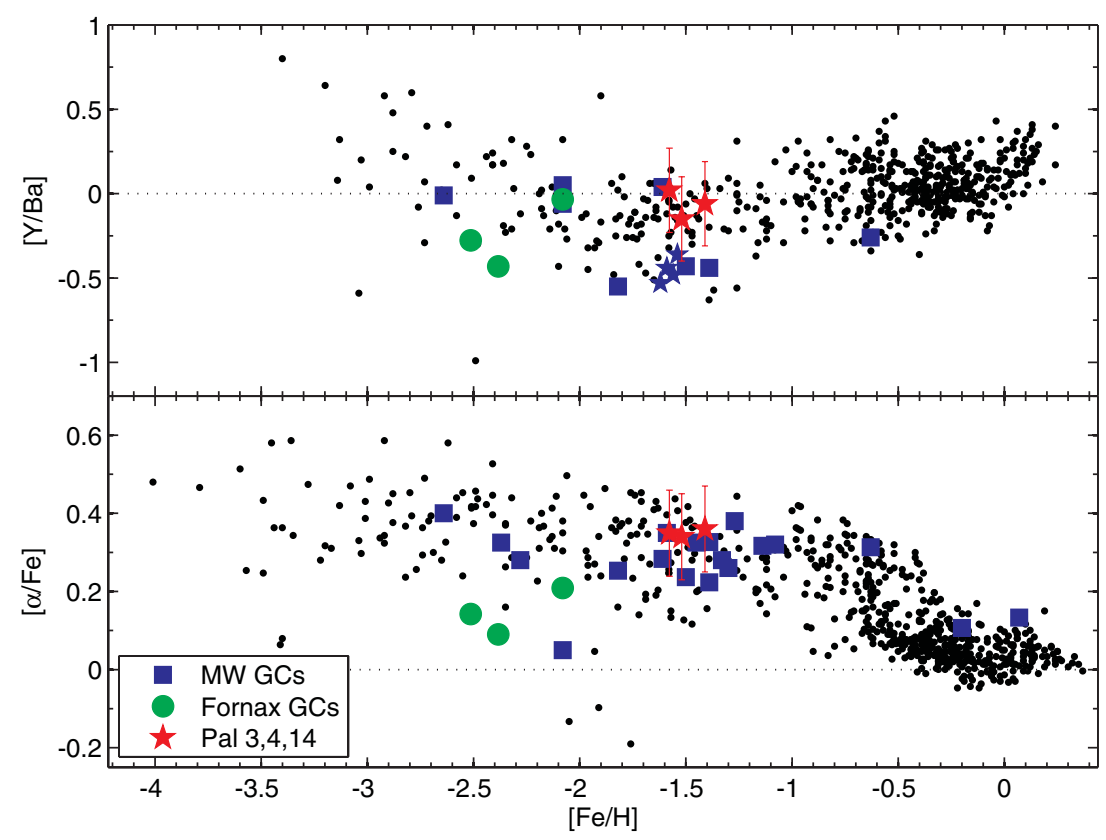

Figure 3. Comparison of the $[\alpha / \mathrm{Fe}]$ abundance ratios in Galactic field stars (black dots), the Fornax dSph ([26]), and MW GCs, with a particular focus on the outer halo clusters Pal 3, 4, and 14 (at $\mathrm{R}_{\mathrm{GC}}=96,111$, and $72 \mathrm{kpc}$ ). See [22], [27], and [35] for details and references.

[20]) - present-day instruments like the VLT/FLAMES multiobject spectrograph can accommodate more than 100 fibres across a field of view of $\sim 25^{\prime}$. This allows us to target stars out to the tidal radius without running out of sources due to crowding, source confusion, or fibre crossings. However, the latter becomes problematic for the remote systems - one has to bear in mind that even the remarkably large radial extent of these GCs translates into a mere few minutes of arc on the sky, given their large distances. In the case of the outer halo GC Pal $3\left(R_{\odot}=93 \mathrm{kpc}\right)$, half-light and tidal radii correspond to 0.4 ' and 4', respectively ([21]), rendering multi-object approaches an inefficient strategy.

Secondly, the (often sparse) red giant branches of the remote GCs are faint and high-resolution spectroscopy requires long exposure times at $\geq 6-m$ class telescopes $\left([22]^{5} ;[23]\right)$. An alternative path to determining chemical abundance ratios for the faint residents of the outer halo is then to obtain integrated cluster spectra ([24]) or to co-add low S/N spectra of many stars to extract a mean abundance information ([22]; [25]). The abundance spread can then be evaluated in a statistical manner. In Fig. 3 we highlight the current knowledge of the chemical inventory of the outer halo GCs in comparison with halo field stars and some archetypical inner halo GCs $\left(\mathrm{R}_{\mathrm{GC}} \lesssim 12 \mathrm{kpc}\right)$. This is done for the $[\alpha / \mathrm{Fe}]$ and $[\mathrm{Y} / \mathrm{Ba}]$ abundance ratios - important tracers of the chemical evolution of any stellar system (e.g., [9]). As already discussed in [22] and [25], these systems do not stand out in any (chemical) regard, safe for a dominance of r-process nucleosynthesis ([22], [27]). For instance, the $[\alpha / \mathrm{Fe}]$ and other heavy element abundance patterns are fully compatible with those in the inner halo, indicating that the inner and outer MW halos have evolved, at least chemically, homogeneously - which contrasts their otherwise diverse characteristics such as a younger age and larger spatial extent compared to the inner systems. This is not self evident as individual systems at large distances show anomalies that question their status

\footnotetext{
5 This work contained individual spectra down to $\mathrm{V}=18.5 \mathrm{mag}$, for which integration times of $\sim 4$ hours were required to reach moderate $\mathrm{S} / \mathrm{N}$ ratios of at least 30 for a handful of stars.
} 


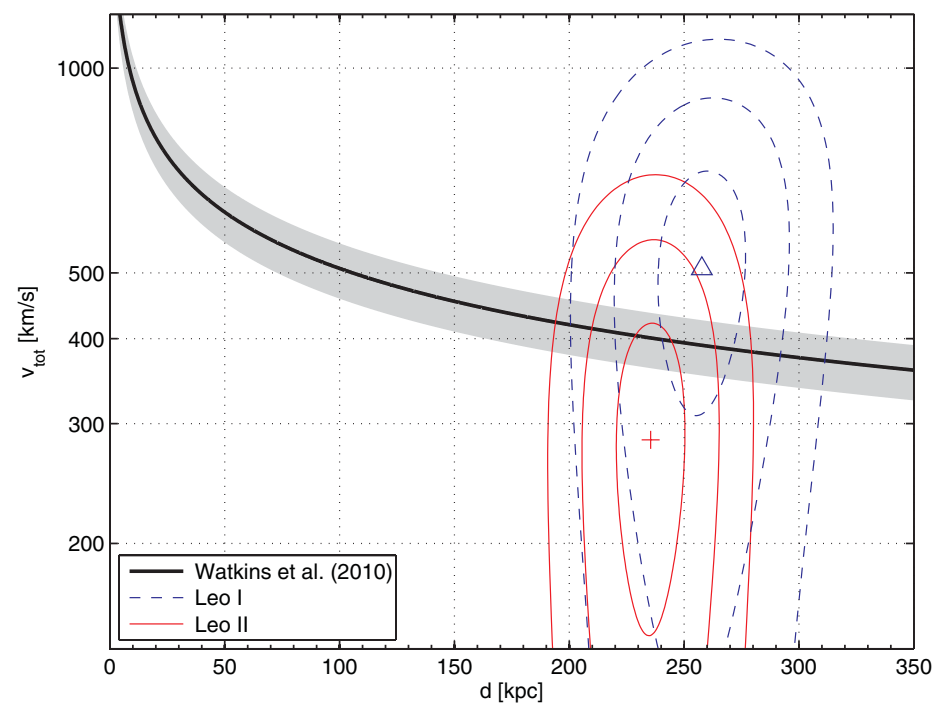

Figure 4. Space velocity of Leo II ([33]) and a preliminary measurement for Leo I (Lépine et al., in prep.) in comparison with the Galactic escape velocity ([31]). Shown each are 1, 2, and $3 \sigma$-contours.

as genuine halo GCs and rather suggests an accretion origin ([23], [28]). While the occurrence of an abundance spread cannot be excluded for at least one remote, extended GC, NGC 2419 ([29]), all other studied systems beyond $\sim 70 \mathrm{kpc}$ do not show any signs of any such variations. Overall, the outer halo population appears dissimilar from the dSph stars, while similarities with GCs within a dSph remain, as the example of the Fornax GCs shows, albeit at metallicities lower by 1 dex ([26]).

\section{OUTER HALO SATELLITES - THE PROPER MOTIONS OF LEO I AND II}

As discussed above, a spectroscopic characterization of the remotest halo structures is feasible (e.g., [30]), yet very (exposure-) time consuming. Another way to tackle the question of a halo assembly is to study the dynamics of satellites; this point of view rather sheds light on the aspect of how much mass needs to be assembled onto the host system to end up with a halo as is observed and what the relative importance of individual, present-day satellites will be. For instance, the recent study of [31] has employed the kinematics of discrete tracers (field stars, GCs, dSphs) to estimate the total mass of the MW out to $300 \mathrm{kpc}$ as $(2.5 \pm 0.5) \times 10^{12} \mathrm{M}_{\odot}$. This procedure is, however, sensitive to the in- or exclusion of kinematic outliers, such as the Leo I and Hercules dSphs with their large distances and relatively high radial velocities (in the Galactic rest frame), which can alter the mass estimator by as much as $\sim 25 \%$. Knowledge of the full phase-space information, in particular the proper motions, of the tracers is required to ultimately construct a realistic mass model for the MW and to assess the membership of any such system with the MW.

At their large distances of 230-250 kpc, proper motion measurements for the Leo I and II dSphs are strictly not any "easier" than obtaining high S/N, high-resolution spectroscopy for their faint stars, as one is chiefly dealing with sub-pixel motions ([32]). Generally, at $100 \mathrm{kpc}$ a transverse velocity of $100 \mathrm{~km} \mathrm{~s}^{-1}$ corresponds to a proper motion of $\sim 0.2 \mathrm{mas} \mathrm{yr}^{-1}$, or a mere $0.03 \mathrm{HST} /$ WFPC2 pixels over a typical base line of 15 years.

In fact, based on 14 years worth of archival HST data, anchored to a system of 17 extragalactic reference sources, we succeeded in determining the proper motion of the remote Leo II dSph (see [33] for details and numbers). The resulting, large space velocity in the Galactic rest frame $\left(v_{\mathrm{GRF}}=\right.$ $\left.266 \pm 129 \mathrm{~km} \mathrm{~s}^{-1}\right)$ is chiefly dominated by a large tangential component $\left(v_{\mathrm{t}}=265 \pm 129 \mathrm{~km} \mathrm{~s}^{-1}\right)$, 
indicating that Leo II is currently at apo- or pericenter, or on a highly eccentric orbit. The comparison with the local MW escape velocity (Fig. 4) indicates that this object is currently formally bound to the Galaxy at the $1 \sigma$-level; at the large distance of these tracers, our assessment is insensitive to the exact choice of the MW potential. On the other hand, the implied "orbital" period amounts to 50 Gyr and its "apocenter" lies well outside $2 \mathrm{Mpc}$, which prohibits us to trace its exact orbital paths unless the entire Local Group's dynamic was accounted for. We conclude that Leo II has rather evolved in isolation (in concordance with its star formation history; [34]) and is now passing through the MW halo for the first time, as is seen also in M31 (e.g., [5]).

While the $8 \%$ fractional contribution of Leo II to the mass budget of the MW ([31]) does not appear pivotal, the role of Leo I (at 27\%) is of prime importance. From a comparable HST data set we were able to measure a proper motion for the latter and the resulting, preliminary space velocity (Fig. 4) implies that Leo I might not be bound to the MW, although this result is marginal at present (at $0.5 \sigma$ ) and needs to await consolidation from our careful analysis (Lépine et al. in prep.). It is likely, that also this dSph has formed and evolved in isolation and is now approaching its first encounter with the (outer) halo of the Galaxy. Whether suchlike objects will actually shed enough stars to contribute significantly to the halo field star population is, however, questionable and needs further orbital study.

\section{References}

[1] Searle, L., \& Zinn, R., ApJ 225, (1978) 357

[2] Zucker, D.B., et al., ApJL 643, (2006) 103

[3] Belokurov, V., et al., ApJ 654, (2007) 897

[4] Walsh, S.M., Jerjen, H., \& Willman, B., ApJ 662, (2007), L83

[5] Majewski, S.R., et al., ApJL 670, (2007) 9

[6] McConnachie, A.W., et al., ApJ 688, (2008) 1009

[7] Koch, A., AN 330, (2009) 675

[8] Misgeld, I., \& Hilker, M., MNRAS 414, (2011) 3619

[9] Tolstoy, E., Hill, V., \& Tosi, M., ARA\&A 47, (2009) 371

[10] Grebel, E.K., Rev. Mod. Ast. 10, (1997) 29

[11] Bovill, M.S., \& Ricotti, M., ApJ 693, (2009) 1859

[12] Harris, W.E., AJ 112, (1996) 1487

[13] Carretta, E., Bragaglia, A., Gratton, R., D’Orazi, V., \& Lucatello, S., A\&A 508, (2009) 695

[14] Carretta, E., et al., A\&A 516, (2010) 55

[15] Niederste-Ostholt, M., et al., MNRAS 398, (2009) 1771

[16] Simon, J.D, et al., ApJ 733, (2011) 46

[17] Norris, J.E., et al., AJ 723, (2010) 1632

[18] Koch, A., et al., ApJ 690, (2009a) 453

[19] Martin, N., de Jong, J.T.A., \& Rix, H.-W., ApJ 684, (2008) 1075

[20] Lind, et al., A\&A 527, (2011) 148

[21] Hilker, M., A\&A 448, (2006) 171

[22] Koch, A., Côté, P., \& McWilliam, A., A\&A 506, (2009b) 729

[23] Cohen, J., Huang, W., \& Kirby, E.N., ApJ 740, (2011) 38

[24] McWilliam, A., \& Bernstein, R., ApJ 684, (2008) 326

[25] Koch, A., \& Côté, P., A\&A 517, (2010) 59

[26] Letarte, B., Hill, V., Jablonka, P., Tolstoy, E., François, P., \& Meylan, G., A\&A 453, (2006) 547

[27] Çalışkan, Ş., Christlieb, N., \& Grebel, E.K., A\&A in press, (2011)

[28] Lee, J.-W., López-Morales, M., \& Carney, B.W., ApJL 646, (2006) 119

[29] Cohen, J.G., Kirby, E.N., Simon, J.D., \& Geha, M., ApJ 725, (2010) 288 
[30] Shetrone, M.D., Siegel, M.H, Cook, D.O., \& Bosler, T.L., AJ 137, (2009) 62

[31] Watkins, L.J., Evans, N.W., \& An., J.H., MNRAS 406, (2010) 264

[32] Anderson, J., \& King, I.R., PASP 112, (2000) 1360

[33] Lépine, S., Koch, A., Rich, R.M., \& Kuijken, K., ApJ 741, (2011) 100

[34] Koch, A., et al., AJ 133, (2007) 270

[35] Koch, A., Adén, D., Grebel, E.K., \& Feltzing, S., IAUS 265, (2010) 227 